\title{
Early Dexamethasone Use as A Protective Measure In Non-Mechanically Ventilated Critically III Patients With COVID-19: A Multicenter, Cohort Study
}

Khalid Al Sulaiman ( $\sim$ alsulaimankh@hotmail.com )

King Abdulaziz Medical City

Ghazwa B. Korayem

Princess Nourah bint Abdulrahman University

Khalid Eljaaly

King Abdulaziz University

Ali F. Altebainawi

King Khalid Hospital, Hail Health Cluster

Omar Al Harbi

King Abdulaziz Medical City

Hisham A. Badreldin

King Saud bin Abdulaziz University for Health Sciences

Abdullah Al Harthi

King Abdulaziz Medical City

Ghada Al Yousif

King Abdulaziz Medical City

Ramesh Vishwakarma

European Organization for Research and Treatment of Cancer (EORTC) Headquarters

Shorouq Albelwi

Princess Nourah bint Abdulrahman University

Rahaf Almutairi

Princess Nourah bint Abdulrahman University

\section{Maha Almousa}

Princess Nourah bint Abdulrahman University

Razan Alghamdi

Princess Nourah bint Abdulrahman University

Alaa Alhubaishi

Princess Nourah bint Abdulrahman University

Abdulrahman Alissa

King Abdullah bin Abdulaziz University Hospital

Aisha Alharbi 
King Abdulaziz University Hospital

\section{Rahmah Algarni}

King Abdulaziz University Hospital

\section{Sarah Al Homaid}

King Saud bin Abdulaziz University for Health Sciences

Khawla Kahtani

King Saud bin Abdulaziz University for Health Sciences

\section{Nada Akhani}

King Fahad Medical City

\section{Abdulaleam Al Atassi}

King Abdulaziz Medical City

Ghassan Al Ghamdi

King Abdulaziz Medical City

\section{Ohoud Aljuhani}

King Abdulaziz University

\section{Research Article}

Keywords: COVID-19, SARS-Cov-2, dexamethasone, steroids, timing, early use, critically ill, Intensive Care Units (ICUs), respiratory failure

Posted Date: November 12th, 2021

DOI: https://doi.org/10.21203/rs.3.rs-1025164/v1

License: (c) (i) This work is licensed under a Creative Commons Attribution 4.0 International License. Read Full License 


\section{Abstract}

Background: Dexamethasone showed mortality benefits in COVID-19 patients. However, the optimal timing for dexamethasone initiation to prevent COVID-19 consequences such as respiratory failure requiring $M V$ is debatable. As a result, the purpose of this study is to assess the impact of early dexamethasone initiation in non-MV critically ill patients with COVID19.

Methods: A multicenter cohort study including adult patients with COVID-19 admitted to intensive care units (ICUs) and received systemic dexamethasone between March 2020 and March 2021. Patients were categorized into two groups based on the timing for dexamethasone initiation (early vs. late). The primary endpoint is developing respiratory failure that required MV; other outcomes were considered secondary. Propensity score matching was used based on the patient's SOFA score, mechanical ventilation (MV) status, prone status, and early use of tocilizumab within 24 hours of ICU admission.

Results: Among 208 patients matched using propensity score, 104 had received dexamethasone after 24 hours of ICU admission (1:1 ratio). Among the non-mechanically ventilated patients, late use of dexamethasone was associated with higher odds of developing respiratory failure that required MV (OR [95\%Cl]: $2.75[1.12,6.76], P=0.02)$. Additionally, late use was associated with longer hospital LOS (Est. [95\% Cl]: 0.55 [0.22, 0.88], $P=0.001)$.The 30-day and in-hospital mortality were higher in the late group; however, were not statistically significant.

Conclusion: Early use of dexamethasone within 24 hours of ICU admission in critically ill patients with COVID-19 might be considered a proactive protective measure in non-mechanically ventilated patients.

\section{Introduction}

In 2019, a newly discovered severe acute respiratory syndrome coronavirus 2 (SARS-CoV-2) was first reported in Wuhan, China.(1) Since the outbreak of this virus, more than 230,000,000 confirmed cases have been reported worldwide.(2) More than 4 million deaths occurred among the confirmed SARS-CoV-2 cases, with overall case mortality of almost $2 \%$ globally.(1) The clinical presentation of COVID-19 ranges in severity from asymptomatic mild illness to severe pneumonia, leading to acute respiratory distress syndrome (ARDS) and associated with a high mortality rate.(3) Severe respiratory symptoms may increase the risk of hospitalization and intensive care unit (ICU) admission.(4) The ICU mortality rate is increased in patients admitted to the ICU and have severe acute lung injury ranging from $26-40 \% .(5,6)$

In critically ill patients with severe COVID-19, the hyperactivation of the systematic inflammatory system causes a state known as "cytokine release syndrome" (CRS).(7) This state may lead to multiple complications such as acute respiratory distress syndrome (ARDS), septic shock, and acute kidney injury (AKI), disseminated intravascular coagulation (DIC), increasing the risk of mortality in those patients. $(1,8$, 9) Currently, several variants of SARS-CoV-2 that cause COVID-19 are discovered, yet there are limited treatment options specific for COVID-19. The mainstay for the treatment of patients with moderate to 
severe COVID-19 is anti-inflammatory/antirheumatic medications, immune-based therapy, antiviral agents, and convalescent plasma.(10)

Given the fact that COVID-19 patients can develop a systemic inflammatory response that can lead to lung injury and multisystem organ dysfunction, corticosteriods (CS) anti-inflammatory effect can serve as a potential therapeutic option. $(11,12)$ The RECOVERY group multicenter, randomized, open-label trial conducted in the United Kingdom showed a significant mortality reduction at 28-days in hospitalized patients who received dexamethasone for up to 10 days compared to patients who received the standard of care.(13) This benefit was observed in patients who were mechanically ventilated or required supplemental oxygen at enrollment.(13) Moreover, a systemic review and meta-analysis including 20,197 patients with COVID-19 reported a significant reduction in mortality and ventilator-free days, the number of patients requiring mechanical ventilation for respiratory failure, and the mechanical ventilator timing.

Although most previous studies showed favorable clinical outcomes and mortality benefits in patients who were initiated CS "early" in the COVID-19 treatment. $(13,15-17)$ the optimal timing for starting CS in critically ill patients with COVID-19 is still being investigated,(18) especially dexamethasones in COVID-19 critically ill patients. Since COVID-19 related lung injury and its associated hyperinflammatory and overreacting immune response occur early in ARDS presentation. We hypothesized that early initiation of dexamethasone could attenuate the inflammatory process early, leading to survival benefits and reduction in further complications. Therefore, this study aims to evaluate the appropriate timing of systemic dexamethasone initiation in critically ill patients with COVID-19 and its clinical outcomes.

\section{Methods}

\section{Study design}

This is a multicenter, non-interventional cohort study including critically ill patients aged $\geq 18$-years with COVID-19 who received dexamethasone and were admitted to the ICU from March 01, 2020, until March 31,2021 . This study was conducted retrospectively and prospectively. The retrospective component included de-identified data of COVID-19 positive patients admitted before the date of IRB approval (March-June 2020). While the prospective component was conducted between July 1, 2020 and March $31,2021$.

Patients were diagnosed with COVID-19 using Reverse Transcriptase-Polymerase Chain Reaction (RTPCR) nasopharyngeal or throat swabs. Eligible patients were then classified into two groups based on the timing of dexamethasone initiation during ICU stay to either "early" or "late." Patients who were initiated on dexamethasone 24 hours of ICU admission were considered in the early group. In contrast, patients who received dexamethasone beyond that time were considered as a late initiation. The study was approved by King Abdullah International Medical Research Center (KAIMRC) in July 2020 (Ref.\# RC20/430/R). All methods were performed in accordance with relevant guidelines and regulations. King 
Abdullah International Medical Research Center (KAIMRC)-IRB committee waived the informed consent due to its retrospective nature.

\section{Settings}

The study was conducted at four hospitals in Saudi Arabia: King Abdulaziz Medical City (Riyadh), King Abdulaziz University Hospital (Jeddah), King Abdullah bin Abdulaziz University Hospital (Riyadh), and King Salman Specialist Hospital (Hail). The primary site for this multicenter study was King Abdulaziz Medical City (Riyadh).

\section{Participants}

All included patients have received a dose of $6 \mathrm{mg}$ IV once daily of dexamethasone based on national and international recommendations. $(13,19)$ Patients were excluded if the dexamethasone was initiated prior to ICU admission, use of dexamethasone interchangeably with methylprednisolone, death within 24 hours of ICU admission, or labeled as "Do-Not-Resuscitate" code status within 24 hours of ICU admission (Figure 1).

\section{Outcomes}

The primary endpoint was to assess association between early dexamethasone initiation and respiratory failure that requires mechanical ventilation in non-MV critically ill patients with COVID-19. The secondary endpoints were 30-day and in-hospital mortality, ICU LOS, hospital LOS, ventilator-free days (VFDs), and complication (s) during ICU stay (i.e., AKI, liver injury, hospital-acquired pneumonia, secondary fungal infection, and thrombosis/ infarction).

\section{Outcome definition (s)}

- Respiratory failure was defined as either low arterial carbon dioxide tension $\left(\mathrm{PaCO}_{2}\right)$ or hypoxemic respiratory failure $\left(\mathrm{PaO}_{2}<60 \mathrm{~mm} \mathrm{Hg}\right.$ with a normal or hypercapnic respiratory failure $\left(\mathrm{PaCO}_{2}>50\right.$ $\mathrm{mm} \mathrm{Hg}$ ) that requires mechanical ventilation.

- The 30-day mortality was defined as the in-hospital death occurring for any cause within 30 days of the admission date during hospital stay.

- Ventilator-free days (VFDs) at 30 days were calculated as following: if the patients die within 30 days of MV, the VFDs $=0$, VFDs $=30$ - days after MV initiation (if patient survived and was successfully liberated from MV), and VFDs $=0$ if the patient is on MV for $>30$ days.

- Acute kidney injury (AKI) was defined as a sudden decrease of renal function within 48 hours, defined by an increase in absolute SCr of at least $26.5 \mu \mathrm{mol} / \mathrm{L}(0.3 \mathrm{mg} / \mathrm{dL})$ or by a percentage increase in $\mathrm{SCr} \geq 50 \%$ (1.5× baseline value) during ICU stay.(20)

- Acute liver injury was defined as alanine aminotransferase (ALT) exceeding three times the upper limit of normal or double in patients with elevated baseline ALT during the ICU stay.

- Secondary fungal infection was identified through the blood, urine, wound, drainage, cerebrospinal fluid (CSF), and/or respiratory cultures. The fungal growth is considered significant if the growth is $\geq$ 
of $100,000 \mathrm{CFU} / \mathrm{ml}$ in sputum or endotracheal aspiration shows; bronchoalveolar lavage (BAL) shows the growth $\geq$ of $10,000 \mathrm{CFU}$ of single organism/ml for protected specimen brushes (PSBs), and $\geq 100,000 \mathrm{CFU}$ of single organism/ml for BAL fluid. Additionally, urinary cultures were considered significant if showing a growth of $\geq 100,000 \mathrm{CFU} / \mathrm{ml}$ of no more than two species of microorganisms. Cultures were excluded if the laboratory reported them as a "contaminant sample". $(21,22)$

- Arterial/venous thrombosis was defined using the International Classification of Diseases, Tenth Revision, Clinical Modification (ICD10-CM) code (i.e., myocardial infarction (MI), ischemic stroke, pulmonary embolism, deep vein thrombosis).(23)

\section{Data collection}

Study data were collected and managed using Research Electronic Data Capture (REDCap $\AA$ ) software hosted by King Abdullah International Medical Research Center (KAIMRC) $(31,32)$. Data collected include patients demographic information (See additional file 1), comorbidities, vital signs and laboratory data, Acute Physiology and Chronic Health Evaluation II (APACHE II), Sequential Organ Failure Assessment (SOFA), and Nutrition Risk in Critically ill (NUTRIC) scores, Glasgow Coma Score (GCS), acute kidney injury, fluid balance, proning position status, the needs for mechanical ventilation (MV) and MV parameters (e.g., $\mathrm{PaO}_{2} / \mathrm{FiO}_{2}$ ratio, $\mathrm{FiO}_{2}$ requirement) within 24 hours of $\mathrm{ICU}$ admission. Also, renal profile, liver function tests (LFTs), coagulation profile (i.e., INR, aPTT, fibrinogen), and inflammatory markers (CRP, procalcitonin) within 24 hours of ICU admission were documented. Tocilizumab use was recorded for the eligible patients. All patients were followed until they were discharged from the hospital or died during hospital stay whichever occurred first.

\section{Statistical analysis}

We presented categorical variables as number (percentage), numerical variables (continuous variables) as mean and standard deviation (SD), or median and lower quartile (Q1) and upper quartile (Q3), as appropriate. The normality assumptions were assessed for all numerical variables using a statistical test (i.e., Shapiro-Wilk test) and graphical representation (i.e., histograms and Q-Q plots). We assessed model fit using the Hosmer-Lemeshow goodness-of-fit test.

We compared categorical variables using the Chi-square or Fisher exact test. We compared the normally distributed continuous variables using the student t-test and other non-normally distributed continuous variables with the Mann-Whitney U test. Baseline characteristics, baseline severity, and outcome variables were compared between the two groups. Model fit assessed using the Hosmer-Lemeshow goodness-of-fit test. We aimed to enroll as many patients as possible, with no predefined sample size. For the primary outcome, non-mechanically ventilated patients were used as the denominator. Multivariable logistic and negative binomial regression analysis were used for the outcomes considered in this study and reported using the odds ratios (OR), or estimates with the $95 \%$ confidence intervals $(\mathrm{Cl})$ as appropriate. Regression analysis was done by considering the PS score as one of the covariates in the model. Additionally, 
Kaplan-Meier (KM) plots were generated for these outcomes. No imputation was made for missing data as the cohort of patients in our study was not derived from random selection. We considered a $P$ value of $<0.05$ statistically significant and used SAS version 9.4 for all statistical analyses.

Propensity score matching procedure (Proc PS match) (SAS, Cary, NC) was used to match patients who received late dexamethasone (active group) to patients who received early dexamethasone (control group) based on patient's SOFA score, mechanical ventilation (MV) status, proning status, and early use of tocilizumab within 24 hours of ICU admission. A greedy nearest neighbor matching method was used in which one patient who received late dexamethasone (active group) matched with one patient who received early dexamethasone (control group), which eventually produced the smallest within-pair difference among all available pairs with treated patients. Patients were matched only if the difference in the logits of the propensity scores for pairs of patients from the two groups was less than or equal to 0.5 times the pooled estimate of the standard deviation.

\section{Results}

\section{Demographic and Clinical Characteristics}

A total of 783 critically ill patients with COVID-19 who received dexamethasone were screened; 487 patients were included during the study period. Out of the 487 patients, $76.4 \%$ (372 patients) received dexamethasone early within 24 hours of ICU admission. A total of 208 patients were included after propensity score matching (1:1 ratio) based on the selected criteria (Figure 1). The majority of the patients were men $(70.1 \%)$, and the mean age of the patients was $62.3 \pm 14.8$ years. The most common comorbidities were diabetes mellitus (61.5\%), hypertension (56.9\%), and dyslipidemia (17.7\%); comorbidities were not significantly different between the two groups except for stroke as presented in Table E1 available in the online supplement.

There was no significant difference between the two groups' baseline severity scores (i.e., APACHE II, and SOFA scores), the needs for MV within 24 hours, baseline Oxygenation Index (OI), PaO2/FiO2 ratio, blood glucose level, lactic acid, platelets count, CRP, Creatine phosphokinase(CPK), ferritin, procalcitonin, eGFR and AKI status within 24 hours of ICU admission after using propensity score. The median APACHE II score was 13 , while the median SOFA score was 4 . Additionally, the difference in early use of tocilizumab within 24 hours of ICU admission was not significant between the two groups, as shown in (Table E1).

\section{Respiratory failure and Mortality}

Among the non-MV critically ill patients with COVID-19, late initation of dexamethasone associated with a higher odds for developing respiratory failure that required MV support (OR $(95 \% \mathrm{Cl}): 2.75(1.12,6.76), P$ $=0.02$ ) as demonstrated in Table 1 . 
Table 1

Regression analysis of complications during ICU admission after Propensity Score

\begin{tabular}{|c|c|c|c|c|c|}
\hline \multirow[t]{2}{*}{ Outcomes } & \multicolumn{2}{|c|}{$\begin{array}{l}\text { n of outcomes/Total no-of } \\
\text { patients }\end{array}$} & \multirow[t]{2}{*}{$\begin{array}{l}\mathrm{P}- \\
\text { value }\end{array}$} & \multirow[t]{2}{*}{$\begin{array}{l}\text { Odds Ratio (OR) } \\
(95 \% \mathrm{Cl})\end{array}$} & \multirow{2}{*}{$\begin{array}{l}\text { P- } \\
\text { value } \\
\$\end{array}$} \\
\hline & Early & Late & & & \\
\hline $\begin{array}{l}\text { Respiratory Failure Required } \\
M V, n(\%) \$^{\star}\end{array}$ & $\begin{array}{l}16 / 40 \\
(40.0)\end{array}$ & $\begin{array}{l}27 / 42 \\
(64.3)\end{array}$ & $0.03^{\wedge \wedge}$ & $2.75(1.12,6.76)$ & 0.02 \\
\hline Acute kidney injury, $\mathrm{n}(\%) \Delta$ & $48(46.2)$ & $43(41.3)$ & $0.48^{\wedge \wedge}$ & $0.82(0.47,1.43)$ & 0.49 \\
\hline Liver injury, $\mathrm{n}(\%) \Delta$ & $11(10.6)$ & $8(7.7)$ & $0.47^{\wedge \wedge}$ & $0.70(0.27,1.83)$ & 0.47 \\
\hline Thrombosis/infarction, $\mathrm{n}(\%) \Delta$ & $6(5.8)$ & $7(6.7)$ & $0.77^{\wedge \wedge}$ & $1.02(0.39,2.67)$ & 0.98 \\
\hline $\begin{array}{l}\text { Hospital acquired pneumonia, } \\
\mathrm{n}(\%) \Delta\end{array}$ & $7(6.7)$ & $10(9.6)$ & $0.45^{\wedge \wedge}$ & $1.49(0.54,4.12)$ & 0.44 \\
\hline $\begin{array}{l}\text { Secondary fungal infection, } \\
\mathrm{n}(\%) \Delta\end{array}$ & $7(9.2)$ & $9(10.5)$ & $0.79^{\wedge \wedge}$ & $1.15(0.41,3.25)$ & 0.79 \\
\hline \multicolumn{6}{|c|}{$\begin{array}{l}\$ * \text { Denominator of the percentage is non-mechanically ventilated patients with } 24 \text { hours of ICU } \\
\text { admission. }\end{array}$} \\
\hline \multicolumn{6}{|c|}{$\Delta$ Denominator of the percentage is the total number of patients } \\
\hline \multicolumn{6}{|c|}{${ }^{\wedge \wedge}$ Chi-square test is used to calculate the P-value. } \\
\hline $\begin{array}{l}\text { \$ Propensity score matched u } \\
\text { admission, proning position, }\end{array}$ & ased on $\mathrm{p}$ & t's SOFA $s$ & $M V n$ & 24 hours of ICU & \\
\hline
\end{tabular}

The mortality within 30 days in early groups was $50.5 \%$ compared with $57.7 \%$ in the late initiation group after using propensity score matching (OR [95\%Cl]: 1.34 [0.77, 2.32], $P=0.29)$. Furthermore, the in-hospital mortality was $51.5 \%$ in the early group versus $62.5 \%$ in the late group, which was higher by 1.6 folds at logistic regression analysis in patients who received dexamethasone after 24 hours of ICU admission (OR [95 percent $\mathrm{Cl}]$ : 1.57 [0.90, 2.74], $\mathrm{P}=0.11$ ); however, it was not statistically significant (Table 2). Additionally, the overall survival probabilities were similar during hospital stay between the two groups after propensity score matched as presented in the survival curve (Figure 2). 
Table 2

Regression analysis of mortality and ICU length of stay after Propensity Score

\begin{tabular}{|c|c|c|c|c|c|}
\hline \multirow[t]{2}{*}{ Outcomes } & \multicolumn{3}{|c|}{$\begin{array}{l}\text { n of outcomes/Total no- } \\
\text { of patients }\end{array}$} & \multirow[t]{2}{*}{$\begin{array}{l}\text { Odds Ratio (HR) } \\
(95 \% \mathrm{Cl})\end{array}$} & \multirow{2}{*}{$\begin{array}{l}P \text { - } \\
\text { value } \\
\$\end{array}$} \\
\hline & Early & Late & $\begin{array}{l}\mathrm{P}- \\
\text { value }\end{array}$ & & \\
\hline 30-day mortality, n (\%) $\Delta$ & $52(50.5)$ & $60(57.7)$ & $0.29^{\wedge \wedge}$ & $1.34(0.77,2.32)$ & 0.29 \\
\hline \multirow[t]{2}{*}{ In-hospital mortality, $\mathbf{n}(\%) \Delta$} & $53(51.5)$ & $65(62.5)$ & $0.11^{\wedge \wedge}$ & $1.57(0.90,2.74)$ & 0.11 \\
\hline & & & & $\begin{array}{l}\text { beta coefficient } \\
\text { (Estimates) }(95 \% \mathrm{Cl})\end{array}$ & $\begin{array}{l}\mathrm{P} \text { - } \\
\text { value } \\
\$\end{array}$ \\
\hline $\begin{array}{l}\text { Ventilator free days, Mean } \\
\text { (SD) }\end{array}$ & $\begin{array}{l}10.8 \\
(13.26)\end{array}$ & $7.7(11.19)$ & $0.08^{\wedge}$ & $-0.31(-1.05,0.43)$ & 0.42 \\
\hline $\begin{array}{l}\text { ICU Length of Stay (Days), } \\
\text { Median }(\mathrm{Q} 1, \mathrm{Q}) \text { \& }\end{array}$ & $\begin{array}{l}7.5(4.00 \\
13.00)\end{array}$ & $\begin{array}{l}12.0(8.00 \\
20.00)\end{array}$ & $0.01^{\wedge}$ & $0.28(-0.06,0.62)$ & 0.11 \\
\hline $\begin{array}{l}\text { Hospital Length of Stay } \\
\text { (Days), Median }(\mathrm{Q} 1, \mathrm{Q} 3)^{\text {\& }}\end{array}$ & $\begin{array}{l}15.0 \\
(10.00 \\
22.00)\end{array}$ & $\begin{array}{l}21.0 \\
(12.00 \\
39.00)\end{array}$ & $0.04^{\wedge}$ & $0.55(0.22,0.88)$ & 0.001 \\
\hline \multicolumn{6}{|c|}{$\Delta$ Denominator of the percentage is the total number of patients } \\
\hline \multicolumn{6}{|c|}{$\&$ Denominator is patients who survived. } \\
\hline \multicolumn{6}{|c|}{ ^ Wilcoxon rank sum test is used to calculate the P-value. } \\
\hline \multicolumn{6}{|c|}{${ }^{\wedge}$ Chi-square test is used to calculate the P-value. } \\
\hline \multicolumn{6}{|c|}{$\begin{array}{l}\text { S* Propensity score adjusted negative binomial regression is used to calculate incidence rate ratio } \\
\text { (IRR) and p-value. }\end{array}$} \\
\hline \multicolumn{6}{|c|}{$\begin{array}{l}\text { \$ Propensity score matched used based on patient's SOFA score, MV within } 24 \text { hours of ICU } \\
\text { admission, proning position, and early use of Tocilizumab within } 24 \text { hours of ICU admission. }\end{array}$} \\
\hline \multicolumn{6}{|c|}{$¥$ Denominator of the percentage is survival. } \\
\hline
\end{tabular}

\section{Complications and length of stay}

Complication during ICU stay such as hospital acquired pneumonia (OR $(95 \% \mathrm{Cl}): 1.49(0.54,4.12)$, $P=0.44)$, secondary fungal infection (OR $(95 \% \mathrm{Cl}): 1.15(0.41,3.25)$, p-value $=0.79), \mathrm{AKI}(\mathrm{OR}(95 \% \mathrm{Cl}): 0.82$ $(047,1.43), P=0.49)$, liver injury (OR $(95 \% \mathrm{Cl}): 0.70(0.27,1.83), P=0.47)$ and thrombosis (OR $(95 \% \mathrm{Cl}): 1.02$ $(0.39,2.67), P=0.98)$ were not statistically significant between the two groups as shown in Table 1. 
Among survived patients who received late dexamethasone, we observed a significantly longer hospital LOS compared to the early initiation (est. [95\% Cl]: 0.55 [0.22, 0.88], $P=0.001$ ). Moreover, late initiation of dexamethasone was associated with significantly fewer VFD (est. [95\%Cl]: -0.31 [-1.05, 0.43], $P=0.42$ ); however did not reach the statistical significance (Table 2).

\section{Follow-up inflammatory markers during stay}

Most of the follow-up inflammatory markers during stay (i.e. D-dimer and CPK) were the same between the two groups, except that ferritin peak level was significantly higher in patients who received dexamethasone after 24 hours of ICU admission in comparison to the early initiation with a beta coefficient $(95 \% \mathrm{Cl}): 0.33(0.01,0.65)$, p-value $<0.04$ (Table 3).

Table 3

Regression analysis of peak inflammatory markers after Propensity Score

\begin{tabular}{|c|c|c|c|c|c|}
\hline \multirow[t]{2}{*}{ Outcomes } & \multicolumn{2}{|c|}{$\begin{array}{l}\text { n of outcomes/Total } \\
\text { no-of patients }\end{array}$} & \multirow{2}{*}{$\begin{array}{l}\mathrm{P}- \\
\text { value } \\
\wedge\end{array}$} & \multirow{2}{*}{$\begin{array}{l}\text { beta coefficient } \\
\text { (Estimates) } \\
(95 \% \mathrm{Cl})\end{array}$} & \multirow{2}{*}{$\begin{array}{l}\mathrm{P} \text { - } \\
\text { value } \\
\$\end{array}$} \\
\hline & Early & Late & & & \\
\hline $\begin{array}{l}\text { Ferritin level follow-up (Peak), Mean } \\
(\text { (SD)) } \Delta\end{array}$ & $\begin{array}{l}1616.5 \\
(1333.75)\end{array}$ & $\begin{array}{l}2250.7 \\
(4883.26)\end{array}$ & 0.37 & $0.33(0.01,0.65)$ & 0.04 \\
\hline $\begin{array}{l}\text { D-dimer level follow-up (Peak), Mean } \\
\text { (SD) } \Delta\end{array}$ & $\begin{array}{l}4.7 \\
(13.30)\end{array}$ & $3.5(6.33)$ & 0.64 & $-0.22(-0.83,0.39)$ & 0.48 \\
\hline $\begin{array}{l}\text { Creatine phosphokinase(CPK) level } \\
\text { follow-up (Peak), Mean (SD) } \Delta\end{array}$ & $\begin{array}{l}1132.8 \\
(4711.68)\end{array}$ & $\begin{array}{l}924.4 \\
(2188.64)\end{array}$ & 0.76 & $-0.29(-0.73,0.14)$ & 0.19 \\
\hline \multicolumn{6}{|c|}{$\begin{array}{l}\$ * \text { Denominator of the percentage is non-mechanically ventilated patients with } 24 \text { hours of ICU } \\
\text { admission. }\end{array}$} \\
\hline \multicolumn{6}{|c|}{$\Delta$ Denominator of the percentage is the total number of patients } \\
\hline \multicolumn{6}{|c|}{ ^ Wilcoxon rank sum test is used to calculate the P-value. } \\
\hline $\begin{array}{l}\text { \$ Propensity score matched used ba } \\
\text { admission, proning position, and eal }\end{array}$ & & & & $\begin{array}{l}24 \text { hours of ICU } \\
\text { f ICU admission. }\end{array}$ & \\
\hline
\end{tabular}

\section{Discussion}

Our study aimed to assess the time of dexamethasone initiation on the clinical outcomes of critically ill patients with COVID-19. A total of 487 patients were included in the analysis; dexamethasone was initiated early in most of the included patients $(76.4 \%)$. This study included patients before releasing RECOVERY Collaborative group study results about dexamethasone use.(13) During that time, some clinicians were hesitant to start dexamethasone early in all patients due to conflicting evidence about steroids. After propensity score matching using the SOFA score, MV status within 24 hours of ICU admission, proning position status, and tocilizumab use within 24 hours of ICU admission, we found that dexamethasone early initiation was associated with lower respiratory failure that required MV support. Additionally, early initiation was associated with shorter hospital LOS among the survived patients. 
In critically ill patients with COVID-19, the dysregulated inflammatory immune response observed can be counteracted by the use of CS to down-regulate the inflammatory immune response and accelerate disease resolution. $(24,25)$ Although the World Health Organization (WHO) initially did not recommend using CS for COVID-19 treatment, as of September 02, 2020, the WHO and the National Institute of Health $(\mathrm{NIH})$ recommended using systemic CS in critically ill patients with severe COVID-19. $(26,27)$ Moreover, the use of dexamethasone is recommended by the surviving sepsis guideline for patients with severe COVID-19 requiring MV and patients with refractory shock. (28) However, none of these guidelines recommend the appropriate time for CS initation in patients with COVID-19.

In our study, early dexamethasone initiation was associated with a significant reduction in respiratory failure that required MV support among non-MV patients. Besides the mortality benefits that were observed with dexamethasone use, limited studies investigated the impact of late CS initiation on respiratory failure that required MV. Even though the $\mathrm{NIH}$ guideline recommends using $\mathrm{CS}$ in severely ill COVID-19 patients who require MV, our study found that early initiation of dexamethasone was associated with a lower odds of respiratory failure that required $M V$ in non-MV patients $(13,26)$. That could be related to dexamethasone's prolonged and potent effect, which can mediate downregulation of systemic and pulmonary inflammation, restore homeostasis, and enhance disease resolution. In parallel to our findings, Monedero et al. reported a lower MV rate in the early steroids group(16) However, in that study, the cutoff of early initiation of CS was at 48 hours from ICU admission while including patients who were started on CS before ICU admission and including patients who received other CS types such as: methylprednisolone.(16)

Our results showed higher 30-day mortality and in-hospital mortality in the late initiation group; yet, were not statistically significant. Previous studies have found mortality benefits when comparing CS use to standard of care alone $(13,14,29)$. In the RECOVERY trial the use of dexamethasone compared to standard of care was associated with lower 28-day mortality among patients hospitalized with COVID19 who received invasive mechanical ventilation or oxygen but not those who didn't need respiratory support.(13) Suggesting that the benefit of dexamethasone in patients with COVID-19 is noticeable receiving dexamethasone more than seven days post-symptoms onset.(13)

Additionally, two meta-analyses and systemic reviews reported a significant reduction in the mortality rates in patients with COVID-19 receiving CS compared to standard care only. $(14,29)$ However, none of these studies assessed the respiratory failure requiring MV, mortality benefit, length of stay (LOS), nor MV duration in the patient who received early dexamethasone to patients who did not. On the other hand, an observational retrospective study including 615 patients with COVID-19 found that starting CS at $>72$ hours from admission associated with significant mortality reduction (HR 0.56,95\% $\mathrm{Cl} 0.38-0.82 ; \mathrm{p}=$ 0.003) compared to earlier initiation of CS (within 24 hours). (30) It is noteworthy that most of the study patients received methylprednisolone (87\%).(30).

We believe that our multicenter non-interventional cohort study is one of the first studies to highlight the appropriate time of dexamethasone initiation and its effects on the clinical outcomes of critically ill 
patients with COVID-19. It is prospective component allows to prospectively explore the association between the time of dexamethasone therapy initiation in COVID19 patients with ICU mortality.

Additionally, it had a predefined cutoff margin of early vs. late initiation time, and it assessed several important clinical outcomes in the final analysis. Nevertheless, we also determined some limitations to our study. First, the observational nature of the study design limits the exclusion of missing data of some variables. Second, despite propensity score matching, some residual confounding factors are still possible. Lastly, there was a dynamic change in the national and international COVID-19 management guidelines as more evidence emerged, affecting the general practice.

\section{Conclusion}

Early use of dexamethasone within 24 hours of ICU admission in COVID-19 critically ill patients might be considered a proactive protective measure in non-MV critically ill patients with COVID-19 to minimize the need for mechanical ventilation. Further randomized clinical and interventional studies are needed to confirm the optimal timing for CS initiation, and its benefit in non-MV COVID-19 critically ill patients.

\section{Declarations}

\section{Acknowledgements}

Not applicable.

\section{Author contributions}

All authors contributed to data collections, analysis, drafted, revised, and approved the final version of the manuscript.

\section{Funding}

None.

\section{Availability of data and material}

The datasets used and/or analyzed during the current study are available from corresponding author on reasonable request.

\section{Ethics approval and consent to participate}

The study was approved in July, 2020 by King Abdullah International Medical Research Center Institutional Review Board, Riyadh, Saudi Arabia (Reference No: RC20/430/R). King Abdullah International Medical Research Center (KAIMRC)-IRB committee waived the informed consent due to the research's method as per the policy of the governmental and local research center. All methods were performed in accordance with relevant guidelines and regulations. Participants' confidentiality was 
strictly observed throughout the study by using anonymous unique serial number for each subject and restricting data only to the investigators.

\section{Consent for publication}

Not applicable.

\section{Competing interests}

No author has a conflict of interest in this study.

\section{References}

1. Guan W, Ni Z, Hu Y, Liang W, Ou C, He J, Liu L, Shan H, Lei C, Hui DSC, Du B, Li L, Zeng G, Yuen K-Y, Chen R, Tang C, Wang T, Chen P, Xiang J, Li S, Wang J, Liang Z, Peng Y, Wei L, Liu Y, Hu Y, Peng P, Wang J, Liu J, et al. Clinical Characteristics of Coronavirus Disease 2019 in China. New England Journal of Medicine 2020;382:1708-1720.

2. World Health Organization. WHO Coronavirus (COVID-19) Dashboard. at <https://covid19. who.int/>.

3. Wu Z, McGoogan JM. Characteristics of and Important Lessons From the Coronavirus Disease 2019 (COVID-19) Outbreak in China. JAMA 2020;323:1239.

4. Grasselli G, Zangrillo A, Zanella A, Antonelli M, Cabrini L, Castelli A, Cereda D, Coluccello A, Foti G, Fumagalli R, lotti G, Latronico N, Lorini L, Merler S, Natalini G, Piatti A, Ranieri MV, Scandroglio AM, Storti E, Cecconi M, Pesenti A, Agosteo E, Alaimo V, Albano G, Albertin A, Alborghetti A, Aldegheri G, Antonini B, Barbara E, et al. Baseline Characteristics and Outcomes of 1591 Patients Infected With SARS-CoV-2 Admitted to ICUs of the Lombardy Region, Italy. JAMA 2020;323:1574.

5. Zhou F, Yu T, Du R, Fan G, Liu Y, Liu Z, Xiang J, Wang Y, Song B, Gu X, Guan L, Wei Y, Li H, Wu X, Xu J, Tu S, Zhang Y, Chen H, Cao B. Clinical course and risk factors for mortality of adult inpatients with COVID-19 in Wuhan, China: a retrospective cohort study. The Lancet 2020;395:1054-1062.

6. al Sulaiman KA, Aljuhani O, Eljaaly K, Alharbi AA, al Shabasy AM, Alsaeedi AS, al Mutairi M, Badreldin HA, al Harbi SA, al Haji HA, al Zumai OI, Vishwakarma RK, Alkatheri A. Clinical features and outcomes of critically ill patients with coronavirus disease 2019 (COVID-19): A multicenter cohort study. International Journal of Infectious Diseases 2021;105:180-187.

7. Coomes EA, Haghbayan H. Interleukin-6 in COVID-19: A systematic review and meta-analysis. medRxiv 2020;doi:10.1101/2020.03.30.20048058.

8. Yang X, Jin Y, Li R, Zhang Z, Sun R, Chen D. Prevalence and impact of acute renal impairment on COVID-19: a systematic review and meta-analysis. Critical Care 2020;24:356.

9. Ahmad I, Rathore FA. Neurological manifestations and complications of COVID-19: A literature review. Journal of Clinical Neuroscience 2020;77:8-12.

10. National Institutes of Health. NIH COVID-19 Treatment Guidelines: Pharmacologic Interventions. at $<$ https://www.covid19treatmentguidelines.nih.gov/management/critical-care/pharmacologic- 
interventions/>.

11. Arabi YM, Chrousos GP, Meduri GU. The ten reasons why corticosteroid therapy reduces mortality in severe COVID-19. Intensive Care Medicine 2020;46:2067-2070.

12. Pujari R, Thommana M v, Ruiz Mercedes B, Serwat A. Therapeutic Options for COVID-19: A Review. Cureus 2020;doi:10.7759/cureus.10480.

13. RECOVERY Collaborative Group. Dexamethasone in Hospitalized Patients with Covid-19. New England Journal of Medicine 2021;384:693-704.

14. van Paassen J, Vos JS, Hoekstra EM, Neumann KMI, Boot PC, Arbous SM. Corticosteroid use in COVID-19 patients: a systematic review and meta-analysis on clinical outcomes. Critical Care 2020;24:696.

15. Hyun JH, Kim MH, Sohn Y, Cho Y, Baek YJ, Kim JH, Ahn JY, Choi JY, Yeom JS, Ahn MY, Kim EJ, Baek $\mathrm{J}-\mathrm{H}, \mathrm{Kim} \mathrm{YK}$, Choi H, Jeong SJ. Effects of early corticosteroid use in patients with severe coronavirus disease 2019. BMC Infectious Diseases 2021;21:506.

16. Monedero P, Gea A, Castro P, Candela-Toha AM, Hernández-Sanz ML, Arruti E, Villar J, Ferrando C. Early corticosteroids are associated with lower mortality in critically ill patients with COVID-19: a cohort study. Critical Care 2021;25:2.

17. Fadel R, Morrison AR, Vahia A, Smith ZR, Chaudhry Z, Bhargava P, Miller J, Kenney RM, Alangaden G, Ramesh MS, Nauriyal V, Lakshmikanth J, Abdul Hamed A, Nadeem O, Griebe K, Johnson JM, Bradley P, Uduman J, Hegab S, Swiderek J, Godfrey A, Jennings J, Gardner-Gray J, Ackerman A, Lezotte J, Ruhala J, Samuel L, Tibbetts RJ, Brar I, et al. Early Short-Course Corticosteroids in Hospitalized Patients With COVID-19. Clinical Infectious Diseases 2020;71:2114-2120.

18. Timing of Corticosteroids in COVID-19. at <https://clinicaltrials.gov/ct2/show/NCT04530409>.

19. Saudi Ministry of Health. Saudi MoH Protocol for Patients Suspected of/Confirmed with COVID-19: Supportive care and antiviral treatment of suspected or confirmed COVID-19 infection (version 2.0) June 17th. 2020. at <https://covid19.cdc.gov.sa>.

20. Lin C-Y. Acute kidney injury classification: AKIN and RIFLE criteria in critical patients. World Journal of Critical Care Medicine 2012;1:40.

21. Aleidan FAS, Alkhelaifi $\mathrm{H}$, Alsenaid A, Alromaizan $\mathrm{H}$, Alsalham F, Almutairi A, Alsulaiman $\mathrm{K}$, Abdel Gadir AG. Incidence and risk factors of carbapenem-resistant Enterobacteriaceae infection in intensive care units: a matched case-control study. Expert Review of Anti-infective Therapy 2021;19:393-398.

22. Koenig SM, Truwit JD. Ventilator-Associated Pneumonia: Diagnosis, Treatment, and Prevention. Clinical Microbiology Reviews 2006;19:637-657.

23. ICD - ICD-10-CM - International Classification of Diseases, Tenth Revision, Clinical Modification. 2021;

24. Mehta P, McAuley DF, Brown M, Sanchez E, Tattersall RS, Manson JJ. COVID-19: consider cytokine storm syndromes and immunosuppression. The Lancet 2020;395:1033-1034. 
25. Jamilloux Y, Henry T, Belot A, Viel S, Fauter M, el Jammal T, Walzer T, François B, Sève P. Should we stimulate or suppress immune responses in COVID-19? Cytokine and anti-cytokine interventions. Autoimmunity Reviews 2020;19:102567.

26. National Isntitutes of Health. NIH Coronavirus Disease 2019 (COVID-19) Treatment Guidelines. at <m https://www.covid19treatmentguidelines.nih.gov/>.

27. COVID-19 Clinical management: Living Guidance, 25 January 2021. at <https://www.who.int/publications/i/item/WHO-2019-nCoV-clinical-2021-1>.

28. Alhazzani W, Møller MH, Arabi YM, Loeb M, Gong MN, Fan E, Oczkowski S, Levy MM, Derde L, Dzierba A, Du B, Aboodi M, Wunsch H, Cecconi M, Koh Y, Chertow DS, Maitland K, Alshamsi F, BelleyCote E, Greco M, Laundy M, Morgan JS, Kesecioglu J, McGeer A, Mermel L, Mammen MJ, Alexander $\mathrm{PE}$, Arrington A, Centofanti JE, et al. Surviving Sepsis Campaign: guidelines on the management of critically ill adults with Coronavirus Disease 2019 (COVID-19). Intensive Care Medicine 2020;46:854887.

29. Sterne JAC, Murthy S, Diaz J v., Slutsky AS, Villar J, Angus DC, Annane D, Azevedo LCP, Berwanger O, Cavalcanti AB, Dequin P-F, Du B, Emberson J, Fisher D, Giraudeau B, Gordon AC, Granholm A, Green C, Haynes R, Heming N, Higgins JPT, Horby P, Jüni P, Landray MJ, le Gouge A, Leclerc M, Lim WS, Machado FR, McArthur C, et al. Association Between Administration of Systemic Corticosteroids and Mortality Among Critically III Patients With COVID-19. JAMA 2020;324:1330.

30. Bahl A, Johnson S, Chen N-W. Timing of corticosteroids impacts mortality in hospitalized COVID-19 patients. Internal and Emergency Medicine 2021;doi:10.1007/s11739-021-02655-6.

31. PA Harris, R Taylor, R Thielke, J Payne, N Gonzalez, JG. Conde, Research electronic data capture (REDCap) - A metadata-driven methodology and workflow process for providing translational research informatics support, J Biomed Inform. 2009 Apr;42(2):377-81.

32. PA Harris, R Taylor, BL Minor, V Elliott, M Fernandez, L O’Neal, L McLeod, G Delacqua, F Delacqua, J Kirby, SN Duda, REDCap Consortium, The REDCap consortium: Building an international community of software partners, J Biomed Inform. 2019 May 9 [doi: 10.1016/j.jbi.2019.103208]

\section{Figures}




\section{Inclusion:}

- Patients who were admitted to ICU with confirmed COVID-19 by Reverse Transcriptase-Polymerase Chain Reaction (RT-PCR) on nasopharyngeal or throat swabs and received Dexamethasone.

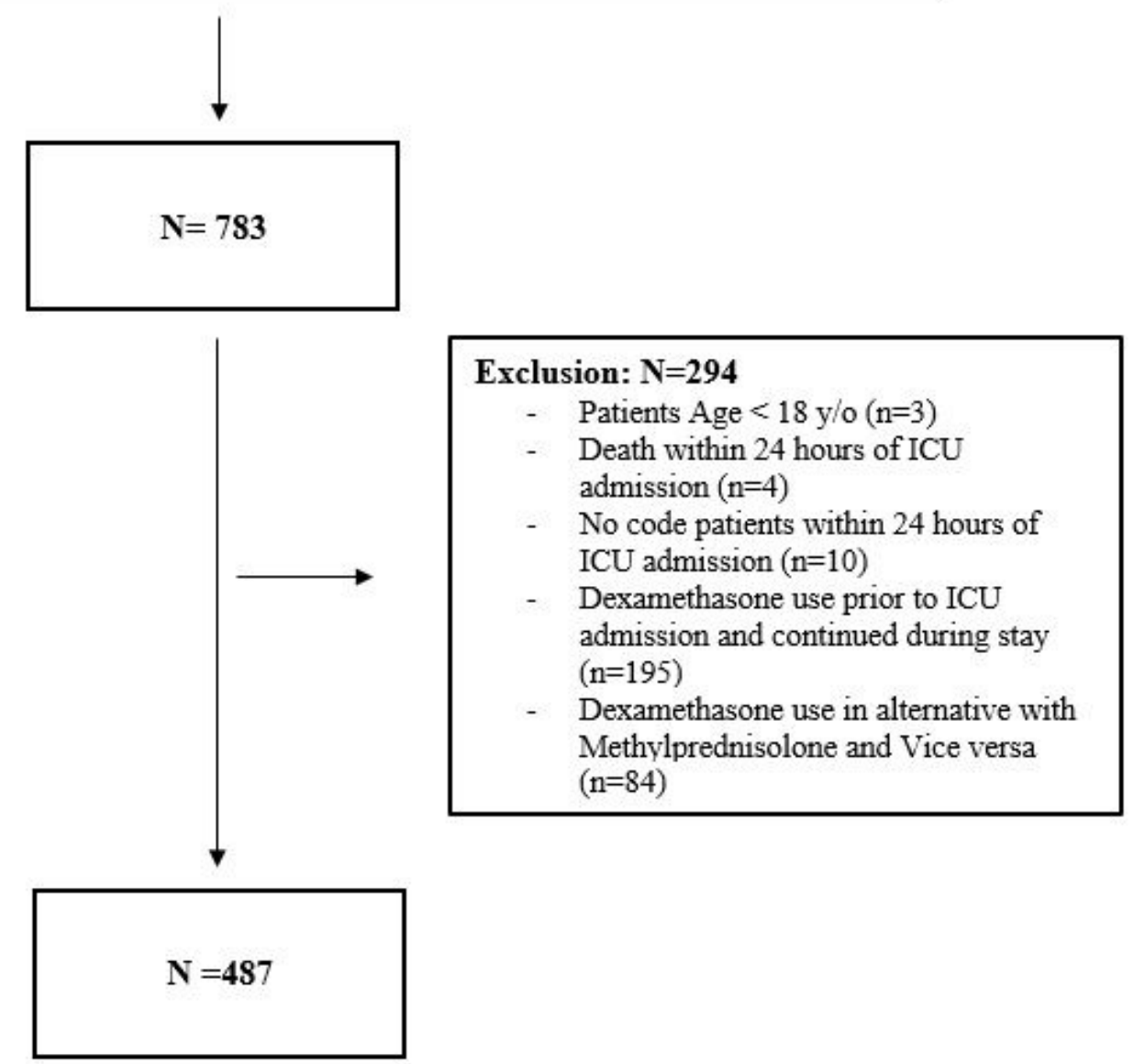

\section{Figure 1}

Flow diagram showing patients recruited with COVID-19 who received dexamethasone 


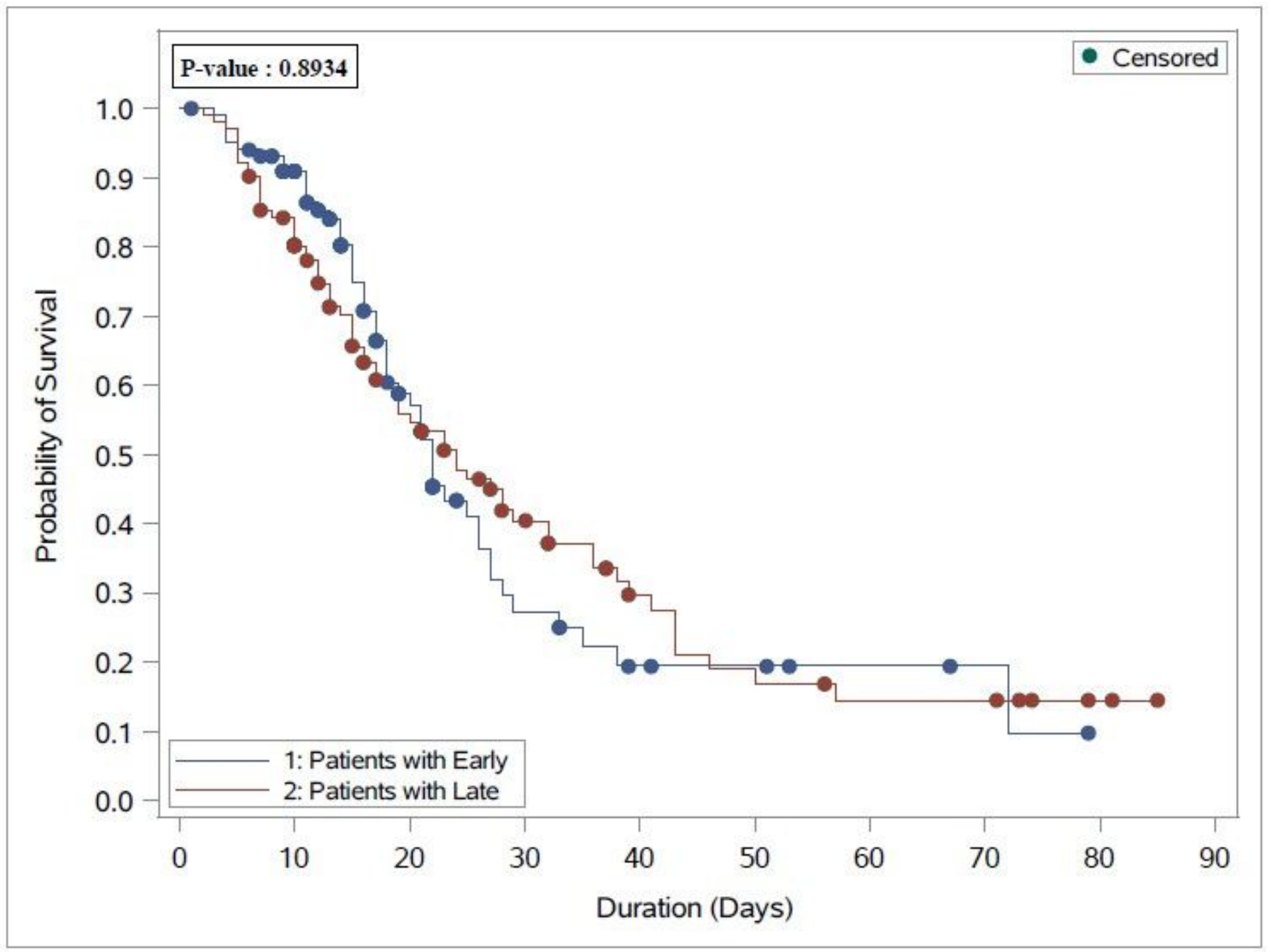

Figure 2

Overall survival plot during the hospital stay after PS matching comparing patients who received late dexamethasone (104 patients) versus the early dexamethasone (104 patients)

\section{Supplementary Files}

This is a list of supplementary files associated with this preprint. Click to download.

- Additionalfile1TableE1.docx 